\title{
Erratum to: A Segmentation Framework of Pulmonary Nodules in Lung CT Images
}

\author{
Ashis Kumar Dhara ${ }^{1}$ - Sudipta Mukhopadhyay ${ }^{1}$ - Rahul Das Gupta ${ }^{2}$ Mandeep Garg ${ }^{3}$. \\ Niranjan Khandelwal ${ }^{3}$
}

Published online: 4 August 2015

(C) Society for Imaging Informatics in Medicine 2015

Erratum to: J Digit Imaging

DOI 10.1007/s10278-015-9801-9

This article inadvertently published with an incomplete author list. The full listing is indicated above.

The online version of the original article can be found at http://dx.doi.org/ 10.1007/s10278-015-9801-9.

Sudipta Mukhopadhyay smukho@gmail.com

1 Department of Electronics and Electrical Communication Engineering, IIT Kharagpur, Kharagpur, India

2 Center for Educational Technology, IIT Kharagpur, Kharagpur, India

3 Department of Radio Diagnosis and Imaging, PGIMER, Chandigarh, India 